This item was submitted to Loughborough's Research Repository by the author.

Items in Figshare are protected by copyright, with all rights reserved, unless otherwise indicated.

\title{
Mapping for nonlinear electron interaction with whistler-mode waves
}

PLEASE CITE THE PUBLISHED VERSION

https://doi.org/10.1063/1.5144477

PUBLISHER

AIP Publishing

VERSION

AM (Accepted Manuscript)

PUBLISHER STATEMENT

This article may be downloaded for personal use only. Any other use requires prior permission of the author and AIP Publishing. This article appeared in Physics of Plasmas, 27 (4), 042902 and may be found at https://aip.scitation.org/doi/abs/10.1063/1.5144477.

\section{LICENCE}

All Rights Reserved

REPOSITORY RECORD

Artemyev, A, Anatoly Neishtadt, and A Vasiliev. 2020. "Mapping for Nonlinear Electron Interaction with Whistler-mode Waves". Loughborough University. https://hdl.handle.net/2134/11996922.v1. 


\section{Mapping for nonlinear electron interaction with whistler-mode waves.}

A. V. Artemyev, ${ }^{1, \text { a) }}$ A. I. Neishtadt, ${ }^{2, \text { b) }}$ and A. A. Vasiliev ${ }^{3}$

${ }^{1)}$ Institute of Geophysics and Planetary Physics, University of California, Los Angeles, USA.

${ }^{2)}$ Department of Mathematical Sciences, Loughborough University, Loughborough LE11 3TU, United Kingdom.

3) Space Research Institute, RAS, Moscow, Russia.

(Dated: 17 March 2020)

The resonant interaction of relativistic electrons and whistler waves is an important mechanism of electron acceleration and scattering in the Earth radiation belts and other space plasma systems. For low amplitude waves, such an interaction is well described by the quasi-linear diffusion theory, whereas nonlinear resonant effects induced by high-amplitude waves are mostly investigated (analytically and numerically) using the test particle approach. In this paper, we develop a mapping technique for the description of this nonlinear resonant interaction. Using the Hamiltonian theory for resonant systems, we derive the main characteristics of electron transport in the phase space and combine these characteristics to construct the map. This map can be considered as a generalization of the classical Chirikov map for systems with nondiffusive particle transport and allows us to model the long-term evolution of the electron distribution function.

\section{INTRODUCTION}

Whistler waves are electromagnetic emissions within the frequency range from the lower-hybrid up to electron cyclotron frequency widely observed in space s. $^{1,47,82,96,98}$ and laboratory ${ }^{71,85}$ plasmas. These waves are generated by various types of electron distributions with thermal anisotropy ${ }^{39,89}$, beam distributions ${ }^{8,43,93,94}$, or $\operatorname{both}^{9,49,52}$, and they play an important role in the isotropisation of originally unstable electron distributions ${ }^{30,45,76}$. A classical theory of whistler wave resonant interaction with electrons is the quasi-linear theory ${ }^{38,46,90}$ that assumes a broad spectrum of low amplitude waves. This theory allows to describe the main characteristics of electron acceleration ${ }^{48,79}$ and scattering 59,80 in the Earth radiation belts, in the solar wind $^{66}$, and at the Earth bow shock ${ }^{91,92}$. However, quasilinear theory cannot describe resonant interactions with the very intense coherent waves ${ }^{34,74}$ often observed in space plasmas ${ }^{2,81,83,95,96,99,100}$. Such sufficiently intense whistlers can resonate nonlinearly with electrons ${ }^{21,35,60}$. Such nonlinear interaction can lead to phase trapping or non-diffusive scattering of particles $s^{5,70}$ and can result in a very fast electron acceleration ${ }^{6,26,27,29,62,75,97}$. Therefore, the effects of nonlinear resonant interaction are actively investigated (see reviews in Refs. 7, 9, 11, 63, and 68).

Since self-consistent Vlasov or Particle-In-Cell simulations of whistler wave generation and their resonances with electrons ${ }^{28,36,44,77}$ can hardly cover the longterm dynamics of the electron distribution in realistic space plasma systems, alternative approaches need to be considered. Beside the test particle approach (i.e., the numerical integration of a large number of electron trajectories ${ }^{3,42,61}$ ), the most interesting approach

\footnotetext{
a) Also at Space Research Institute, RAS, Moscow, Russia; Electronic mail: aartemyev@igpp.ucla.edu

b) Also at Space Research Institute, RAS, Moscow, Russia
}

for the investigation of nonlinear electron resonances with whistler waves consists in the derivation of a kinetic equation (master-equation ${ }^{86}$ ) describing the evolution of the electron distribution. This approach generalizes the quasi-linear diffusion equation by including terms responsible for electron nonlinear acceleration and scattering ${ }^{13,18,29,64}$. Such terms can be derived analytically $^{15,84}$ or numerically ${ }^{31,32,64}$.

A less investigated but potentially useful approach is the mapping technique already widely used for systems with small wave amplitudes ${ }^{50,65}$. The well-known Chirikov map ${ }^{25}$ describes phase space diffusion and transport induced by periodical random jumps of particle momentum. The resonance of electrons and whistler waves results in a similar type of dynamics: each resonant interaction corresponds to an electron energy (and pitch-angle) jump inducing particle transport in phase space. For small amplitude whistler waves, the map of electron resonant jumps is quite similar to the Chirikov map $^{22,40,41}$, but nonlinear resonant interaction should significantly change such a map. In this study, we develop a map describing electron motion in a system with multiple passages through nonlinear resonances. We have also demonstrated that this map models well the electron distribution evolution and can be used to study the radiation belt dynamics.

\section{BASIC EQUATIONS}

To derive the basic properties of the nonlinear electron $\left(m_{e}\right.$ is the rest mass and $-e$ is the charge) interaction with field-aligned whistler waves ( $\omega$ is a constant frequency, $k(\omega, s)$ is the wave number given by the cold plasma dispersion relation ${ }^{72}$ and depending on the fieldaligned coordinate $s$ ) we consider Hamiltonian (see de- 
tails in, e.g., Refs. 16 and 84):

$$
\begin{aligned}
H & =m_{e} c^{2} \gamma+U_{w}\left(s, I_{x}\right) \sin (\phi+\psi) \\
\gamma & =\sqrt{1+\frac{p_{\|}^{2}}{m_{e}^{2} c^{2}}+\frac{2 I_{x} \Omega_{c e}}{m_{e} c^{2}}}
\end{aligned}
$$

where $c$ is the speed of light, $\gamma$ is the gamma factor of the gyroaveraged system, $\Omega_{c e}=e B_{0}(s) / m_{e} c$ is the electron gyrofrequency in the background magnetic field $B_{0}(s)$ given by the reduced dipole $\operatorname{model}^{21}, U_{w}=$ $\sqrt{2 I_{x} \Omega_{c e} m_{e}} e B_{w} / \gamma m_{e} c k$ with $B_{w}$ the wave amplitude, $I_{x}$ is the magnetic moment normalized in a such a way that $I_{x} \Omega_{c e}$ has the dimension of energy. The conjugate pairs of variables in Eq. (1) are field-aligned coordinate and momentum, $\left(s, p_{\|}\right)$, and gyrophase and magnetic moment, $\psi, I_{x}$. Wave phase $\phi$ is given by the differential equation: $\dot{\phi}=k(s) \dot{s}-\omega$ (we omit the argument $\omega$ in the function $k)$. In system (1) phases $\phi, \psi$ change much faster than variables $s, p_{\|}, I_{x}$ because wave frequency $\omega$ and gyrofrequency $\Omega_{c e}$ are much larger than the electron bounce frequency $\sim c / R$ where $R$ is a spatial scale of $B_{0}(s)$ gradient. Therefore, the first small parameter of the system is $c / R \Omega_{c e} \ll 1$. The second small parameter of the system is $B_{w} / B_{0} \ll 1$, and we consider sufficiently intense waves with $B_{w} / B_{0} \geq c / R \Omega_{c e}$ (this condition is satisfied for a significant fraction of whistler waves observed in the Earth radiation belts, see Ref. 100).

Figure 1(a) shows several fragments of electron trajectories around the resonance $\dot{\phi}+\dot{\psi}=0$ (with $\dot{\psi}=$ $\left.\partial H / \partial I_{x} \approx \Omega_{c e} / \gamma\right)$ : there are two main effects ${ }^{57}$ - electron trapping into resonance with the energy increase $\Delta \gamma_{\text {trap }}$, and electron scattering on the resonance with the energy decrease $\Delta \gamma_{\text {scat }}$. We plan to construct a map describing the long-term evolution produced by these two processes.

We start with the determination of $\Delta \gamma_{\text {trap }}, \Delta \gamma_{\text {scat }}$ and their dependencies on particle characteristics. First, we use the generating function $W_{1}=\left(\left(\int k(\tilde{s}) d \tilde{s}-\omega t\right)+\psi\right) I+$ $s P$ to introduce phase $\varphi=\phi+\psi$ and conjugate momentum $I$ :

$$
\begin{aligned}
H_{I} & =-\omega I+m_{e} c^{2} \gamma+U_{w}(s, I) \sin \varphi \\
\gamma & =\sqrt{1+\frac{(P+k I)^{2}}{m_{e}^{2} c^{2}}+\frac{2 I \Omega_{c e}}{m_{e} c^{2}}}
\end{aligned}
$$

Pairs of conjugate variables are $(I, \varphi)$ and $(s, P)$. The resonance condition $\left(\dot{\varphi}=\partial H_{I} / \partial I=0\right)$ for Hamiltonian (2) defines the resonant momentum $I_{R}(s, P)$ :

$$
\begin{aligned}
\frac{k I_{R}}{m_{e} c} & =\sqrt{\frac{1-\left(\Omega_{c e} / k c\right)^{2}-2\left(\Omega_{c e} P / k m_{e} c^{2}\right)}{(k c / \omega)^{2}-1}} \\
& -\frac{\Omega_{c e}}{k c}-\frac{P}{m_{e} c}
\end{aligned}
$$

We expand Hamiltonian (2) around the resonance

$$
\begin{aligned}
H_{I} & \approx \Lambda+\frac{1}{2} g\left(I-I_{R}\right)^{2}+U_{w}\left(s, I_{R}\right) \sin \varphi \\
\Lambda & =-\omega I_{R}+m_{e} c^{2} \gamma_{R}, \quad \gamma_{R}=\sqrt{1+\frac{\left(P+k I_{R}\right)^{2}}{m_{e}^{2} c^{2}}+\frac{2 I_{R} \Omega_{c e}}{m_{e} c^{2}}} \\
g & =\left.m_{e} c^{2} \frac{\partial^{2} \gamma}{\partial I^{2}}\right|_{I=I_{R}}=\frac{\omega^{2}\left((k c / \omega)^{2}-1\right)}{\gamma_{R}}
\end{aligned}
$$

and use the generating function $W_{2}=\left(I-I_{R}\right) \zeta+P s^{*}$ to introduce new pairs of conjugate variables $\left(\zeta, P_{\zeta}\right)$ and $\left(s^{*}, P^{*}\right)$ with $P_{\zeta}=I-I_{R}, s^{*}=s+\left(\partial I_{R} / \partial P\right) \zeta, P^{*}=$ $P-\left(\partial I_{R} / \partial s\right) \zeta:$

$$
\begin{aligned}
\tilde{H}_{I} & =\Lambda\left(s^{*}, P^{*}\right)+\frac{1}{2} g P_{\zeta}^{2}+U_{w}\left(s, I_{R}\right) \sin \zeta \\
& \approx \Lambda(s, P)+\frac{1}{2} g P_{\zeta}^{2}-r \zeta+U_{w}\left(s, I_{R}\right) \sin \zeta \\
r & =\left\{\Lambda, I_{R}\right\}_{s, P}=\frac{\partial \Lambda}{\partial s} \frac{\partial I_{R}}{\partial P}-\frac{\partial \Lambda}{\partial P} \frac{\partial I_{R}}{\partial s}
\end{aligned}
$$

Using Hamiltonian (2), we get $m_{e} c^{2} \Delta \gamma=\omega \Delta I$ (note $\left.\partial H_{I} / \partial t=0\right)$ and $\dot{I}=-\partial H_{I} / \partial \varphi=-U_{w} \cos (\varphi)$. Therefore, the energy change $\Delta \gamma$ due to resonant interaction can be written as ${ }^{11,56}$

$$
\begin{gathered}
m_{e} c^{2} \Delta \gamma=-\omega U_{w} \int_{-\infty}^{+\infty} \cos \varphi d t=-2 \omega U_{w} \int_{t_{R}}^{+\infty} \cos \varphi d t \\
=-2 \omega U_{w} \int_{\zeta_{R}}^{+\infty} \frac{\cos \zeta}{g P_{\zeta}} d \zeta=-\sqrt{\frac{2}{g}} \omega \int_{\zeta_{R}}^{+\infty} \frac{U_{w} \cos \zeta d \zeta}{\sqrt{h_{\zeta}+r \zeta-U_{w} \sin \zeta}} \\
=-\sqrt{\frac{2 U_{w}}{g}} \omega \int_{\zeta_{R}}^{+\infty} \frac{a \cos \zeta d \zeta}{\sqrt{\left(\zeta-\zeta_{R}\right)-a\left(\sin \zeta-\sin \zeta_{R}\right)}} \\
=-\sqrt{\frac{2 r}{g}} \omega f\left(\zeta_{R}, a\right)
\end{gathered}
$$

where $t_{R}$ is the time of the resonant interaction, $\zeta_{R}$ is the value of $\zeta$ at $t=t_{R}$, and we use the Hamiltonian equation $\dot{\zeta}=\partial \tilde{H}_{I} / \partial P_{\zeta}$ to express $P_{\zeta}$ through the energy at the resonance $h_{\zeta}=\tilde{H}_{I}-\Lambda=U_{w} \sin \zeta_{R}-r \zeta_{R}$ (note Eq. (6) is written for $r>0$, see details in Refs. 11 and 56). Coefficient $a=U_{w} / r$ determines the mode of resonant interaction: for $|a|>1$ we deal with nonlinear interaction with $\langle\Delta \gamma\rangle_{h_{\zeta}} \neq 0$, where $\langle\cdot\rangle_{h_{\zeta}}$ denotes averaging with respect to $h_{\zeta}$. Function $f\left(\zeta_{R}, a\right)$ is shown in Fig. 2 (a). This is a periodic function with the average value ${ }^{11,56}$ equal to

$$
\langle\Delta \gamma\rangle_{h_{\zeta}}=\frac{\omega}{\pi} \sqrt{\frac{2 r}{g}} \int_{\zeta_{-}}^{\zeta_{+}} \sqrt{\left(\zeta-\zeta_{-}\right)-a\left(\sin \zeta-\sin \zeta_{-}\right)} d \zeta
$$

where $\zeta_{ \pm}$are shown in the phase portrait of the Hamiltonian $\tilde{H}_{I}-\Lambda$ (see Fig. $1(\mathrm{~b})$ ). 

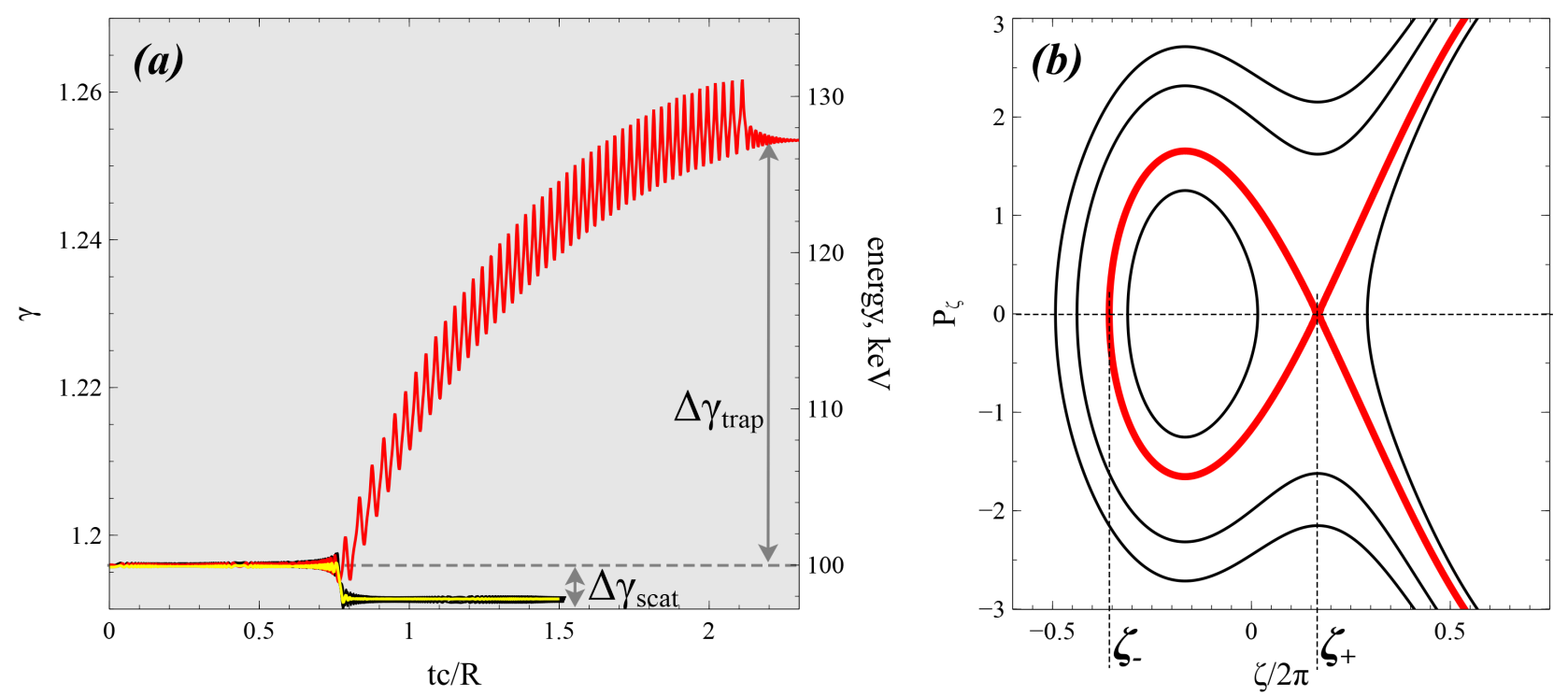

FIG. 1: (a) Change of electron energy due scattering (black; yellow shows averaged energy of scattered particle) and trapping (red). The time interval of one resonant interaction is shown. All electrons have initially the same energy and pitch-angle. For these trajectories and throughout the paper we consider a curvature-free dipole magnetic field ${ }^{21}$ with the radial distance from the Earth $R=4.5$ of the Earth radii. The whistler wave frequency is 0.35 times

the electron cyclotron frequency at the equator, and plasma frequency equals to 4.5 of the electron cyclotron

frequency at the equator. To evaluate the wave number $k$ we use the cold plasma dispersion of whistler waves ${ }^{72}$. Wave amplitude is $300 \mathrm{pT}$, typical for intense whistlers observed in the radiation belts ${ }^{24,83,95,99}$. The distribution of the wave amplitude along magnetic field lines, $B_{w}(s)$, is modeled by function $\tanh \left(\left(\lambda / \delta \lambda_{1}\right)^{2}\right) \exp \left(-\left(\lambda / \delta \lambda_{2}\right)^{2}\right)$ with $\lambda$ the magnetic latitude $\left(d s=R d \lambda \sqrt{1+\sin ^{2} \lambda} \cos \lambda\right)$ and $\delta \lambda_{1}=2^{\circ}, \delta \lambda_{2}=20^{\circ}$. This function fits the observed whistler wave intensity distribution ${ }^{1}$. To simplify the simulations, we consider waves in only one hemisphere, $B_{w}=0$ for $s<0$, and thus there is only one resonance for electrons within one bounce period. Waves are moving away from the equatorial plane, $s=0$, to large $s$, i.e. only $k>0$ are included. (b) Phase portrait of Hamiltonian

$$
\tilde{H}_{I}-\Lambda=g P_{\zeta}^{2} / 2-r \zeta+U_{w} \sin \zeta \text { for }\left|U_{w} / r\right|>1 \text {. }
$$

The energy change in Eq. (7) represents the energy scattering $\Delta \gamma_{\text {scat }}=\langle\Delta \gamma\rangle_{h_{\zeta}}$ and depends on the resonance position $s_{R}$ given by equations

$$
h=-\omega I_{R}(s, P)+m_{e} c^{2} \gamma_{R}(s, P), \quad \gamma_{0}=\gamma_{R}(s, P)
$$

where $m_{e} c^{2} \gamma_{0}$ being the initial electron energy and $h=$ $-\omega I_{x 0}+m_{e} c^{2} \gamma_{0}$ (with $I_{x 0}$ being the initial $I_{x}$ value). Equations (8) can be rewritten as

$$
\gamma_{R}=\left|\frac{\Omega_{c e}}{k c} \mp \frac{k c}{\sqrt{(k c)^{2}-\omega^{2}}} \sqrt{1+\left(\frac{\Omega_{c e}}{k c}\right)^{2}-\frac{2 h}{m_{e} c^{2}} \frac{\Omega_{c e}}{k c}}\right|
$$

Figure 2(b) shows $\Delta \gamma_{\text {scat }}\left(s_{R}\right)$. For a given $h$ (or equivalently for a given initial pitch-angle $\alpha_{0}$ determining $\left.I_{x 0}\right)$ we can plot $\Delta \gamma_{\text {scat }}$ as a function of initial energy $m_{e} c^{2} \gamma_{0}$, see Figure 2(c). As $h$ is determined by $\alpha_{0}$ and $\gamma_{0}$, the energy scattering value $m_{e} c^{2} \Delta \gamma_{s c a t}$ depends on $\alpha_{0}, \gamma_{0}$. Analogous dependencies of scattering on initial particle parameters has been tested for several specific Hamiltonians ${ }^{15,19,84}$. For Hamiltonian (1) we compare the numerically calculated $\Delta \gamma_{\text {scat }}$ with the analytical expression (7) in Fig. 2(c): to evaluate $\Delta \gamma_{\text {scat }}$ numerically, for several $\gamma_{0}$ we run $10^{4}$ trajectories for Hamiltonian (1) for fixed $h$ and different $\gamma_{0}$ (the time of integration of each trajectory includes only one resonant interaction) and then average energy changes.

In contrast to scattering, particle trapping is a nonlocal process. The energy change due to trapping significantly exceeds $\Delta \gamma_{\text {scat }}$ and can be comparable with the initial particle energy. Particles can be trapped if the probability of trapping $\Pi$ is positive ${ }^{11,57}$. For system (5), this probability is defined by the relation ${ }^{20}$ :

$$
\Pi=-\frac{m_{e} c^{2}}{\omega} \frac{d \Delta \gamma_{s c a t}}{d I}=-\frac{m_{e} c^{2}}{\omega}\left\{\Delta \gamma_{s c a t}, I_{R}\right\}
$$

where $\{\cdot, \cdot\}$ is the Poisson bracket with respect to the variables $s, P$. The value $\Pi$ depends on the initial energy $\gamma_{0}$ and $I_{x}$ in terms of their combination $h=-\omega I_{x 0}+$ $m_{e} c^{2} \gamma_{0}$. If Eq. (10) gives a negative value, $\Pi$ should be set to be zero and there are no trapped particles.

Equation (10) determines the relative number of resonant particles that get trapped during a single resonant interaction. Analogous equations have been verified using the test particle calculations for several specific Hamiltonians in Refs. 15, 20, and 84. Note that 

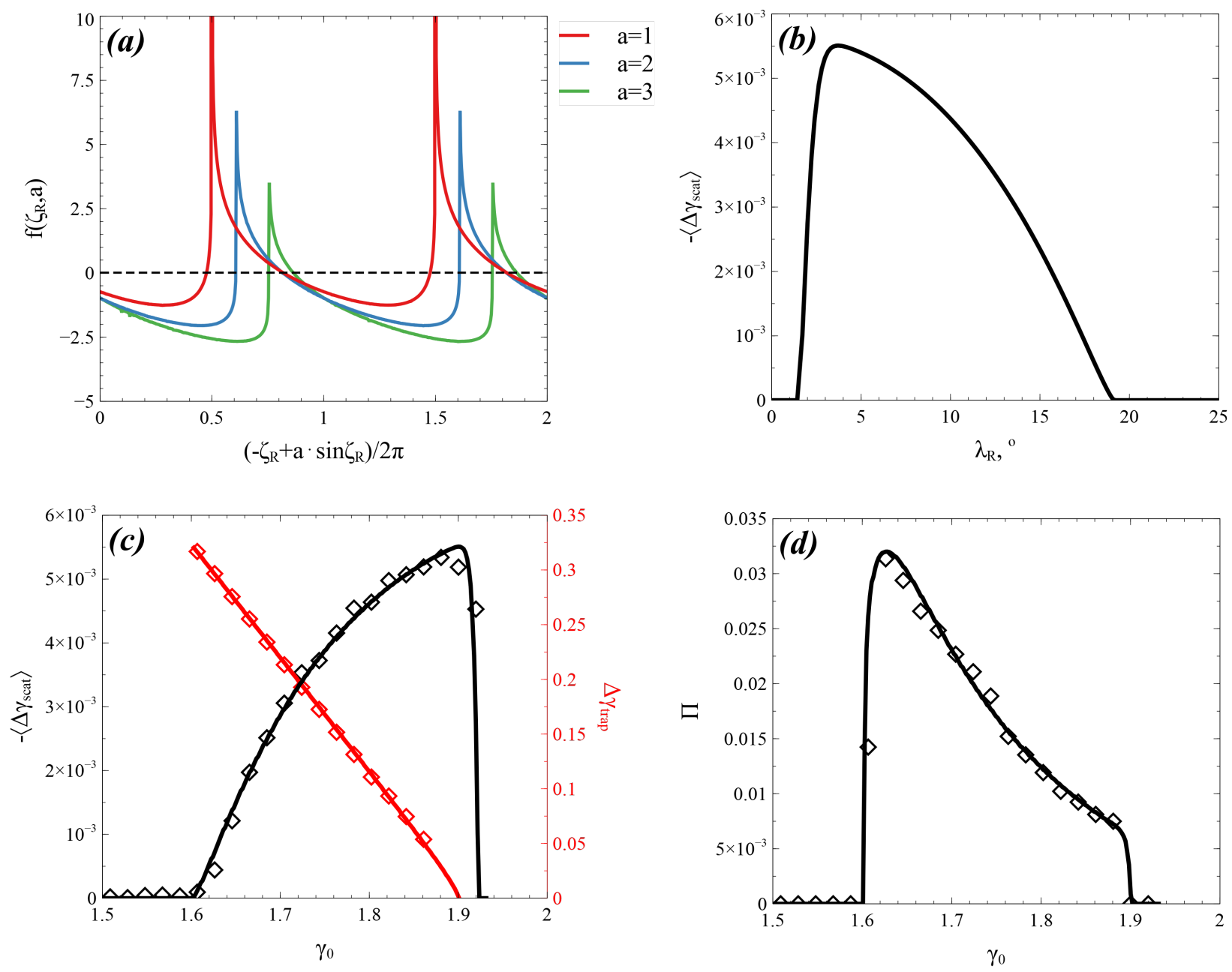

FIG. 2: Main parameters of the resonant system: (a) function $f\left(\zeta_{R}, a\right)$; (b) function $\left\langle\Delta \gamma_{s c a t}\left(\lambda_{R}\right)\right\rangle$, (c) function $\left\langle\Delta \gamma_{\text {scat }}\left(\gamma_{0}\right)\right\rangle$; (d) probability of trapping $\Pi\left(\gamma_{0}\right)$. Symbols show the results of numerical simulations $\left(h / m_{e} c^{2}=1.454\right.$ corresponds to, e.g., $\alpha_{0}=45^{\circ}$ of the equatorial pitch-angle for $300 \mathrm{keV}$ electron energy; see details of model parameters in the caption of Fig. 1).

due to conservation of $h$, the change of $I$ is equal to the change of $\gamma m_{e} c^{2} / \omega$, and thus Eq. (10) can be written as $\Pi=-d \Delta \gamma_{s c a t} / d \gamma$, i.e. the derivative of the profile $\Delta \gamma_{\text {scat }}(\gamma)$ from Fig. 2(c) should give the $\Pi(\gamma)$ profile (for fixed $h$ ). Figure 2(d) shows this $\Pi(\gamma)$ and the corresponding numerical verifications (each numerical point shows the relative amount of $10^{4}$ particles that have been trapped during the first resonant interaction).

Being trapped at some resonant value $s_{R}$ defined by Eqs. (8), particles should escape at $s_{\text {detrap }}$ with an energy gain $\Delta \gamma_{\text {trap }}=\gamma_{R}\left(s_{\text {detrap }}\right)-\gamma_{0}$. This detrapping coordinate can be calculated using the conservation of the adiabatic invariant $(2 \pi)^{-1} \oint P_{\zeta} d \zeta$ for trapped particles (see details in, e.g., Refs. 15 and 84). Formally speaking, $s_{\text {detrap }}$ is the solution of equation $\Delta \gamma_{\text {scat }}\left(s_{\text {detrap }}\right)=$ $\Delta \gamma_{\text {scat }}\left(s_{R}\right)$, i.e., the function (7) should have the same value at the trapping and detrapping positions ${ }^{20,87}$.

To summarize, for a given $h=-\omega I_{x 0}+\gamma_{0} m_{e} c^{2}$, the resonant system (1) can be reduced to a 1D system that is described by the profile of energy change due to scattering $\Delta \gamma_{\text {scat }}(\gamma)$, probability of trapping $\Pi=$ $\max \left(0,-d \Delta \gamma_{s c a t} / d \gamma\right)$, and energy change due to trapping $\Delta \gamma_{\text {trap }}(\gamma)$. These three functions allow us to construct a map describing the system evolution on a time interval including many resonant interactions.

\section{MAPPING TECHNIQUE}

Let us discuss the meaning of the probability of trapping, П. Each trajectory far from the resonance is characterized by initial energy $\gamma$, magnetic moment $I_{x}$, coordinates in the $\left(s, p_{\|}\right)$plane, and phase $\zeta$ which coincides with $\varphi$. Knowing these values we can determine if particles will be trapped or scattered during the first resonant 
interaction. However, particle phase $\zeta$ changes with time much faster than particle $s, p_{\|}$coordinates $\left(\dot{\zeta} \sim \Omega_{c e}\right.$ is the largest frequency in the system). Even a small initial variation of $\zeta$ can result in a crucial change of the particle's fate: trapped particles may become scattered and vice versa. Accordingly, instead of tracing individual trajectories with given $\zeta$, it is more suitable to adopt a probabilistic approach and to consider the relative amount of trapped particle trajectories, equal to $\Pi$ (see Refs. 55 and 67).

Will a particle be trapped or scattered depends on $\zeta$ value at the resonance, $\zeta_{R}$, but instead of $\zeta$ it is more convenient to use the normalized resonant en$\operatorname{ergy} \xi=\left(a \sin \zeta_{R}-\zeta_{R}\right) / 2 \pi-\left(a \sin \zeta_{+}-\zeta_{+}\right) / 2 \pi$ (where $\zeta_{R} \in\left[\zeta_{+}-2 \pi, \zeta_{-}\right]$, see Fig. 1(b)), which is distributed uniformly (see numerical tests of $\xi$ distributions in, e.g., Refs. 17 and 33); its values belong to the interval $[0,1]$. Within this interval, the measure of the sub-range corresponding to trapping equals $\Pi$, and this sub-range is $0 \leq \xi \leq \Pi$. As the particle energy does not change between two successive resonant interactions, we can write a map of the $\gamma \rightarrow \bar{\gamma}$ transition during a single resonance interaction:

$$
\begin{aligned}
& \bar{\gamma}=\gamma+ \begin{cases}\Delta \gamma_{\text {trap }}(\gamma), & \xi \in[0, \Pi) \\
\Delta \gamma_{\text {scat }}(\gamma), & \xi \in(\Pi, 1]\end{cases} \\
& \Pi=-d \Delta \gamma_{\text {scat }} / d \gamma
\end{aligned}
$$

The map (11) should be supplemented with a map for value $\xi$, which is related to $\zeta$ change (gain) between two resonances through the equation (see Eq. (34) in Appendix A):

$$
\bar{\xi}=\xi-\Delta \zeta / 2 \pi \bmod 1
$$

The rate of $\zeta$ change is defined by the Hamiltonian system (2) (note that $\zeta=\varphi$ ), but it is more convenient to use notations of the Hamiltonian system (1):

$$
\begin{gathered}
\dot{\zeta}=-\omega+\frac{\Omega_{c e}(s)}{\gamma}+k(s) \frac{p_{\|}}{m_{e} \gamma} \\
p_{\|}=m_{e} c \sqrt{\gamma^{2}-1-\frac{2 I_{x} \Omega_{c e}(s)}{m_{e} c^{2}}}
\end{gathered}
$$

Integrating Eq. (13) over the time interval between two resonances (in the system under consideration this time is equal to the bounce period $\tau_{b}$ ), we obtain

$$
\begin{aligned}
\Delta \zeta & =\omega \tau_{b}\left(\frac{\varpi}{\gamma}-1\right) \\
\tau_{b} & =\frac{4}{c} \int_{0}^{s_{\max }}\left(\gamma^{2}-1-\frac{2 I_{x} \Omega_{c e}(s)}{m_{e} c^{2}}\right)^{-1 / 2} d s \\
\varpi & =\frac{\int_{0}^{s_{\max }} \Omega_{c e}(s)\left(\gamma^{2}-1-\frac{2 I_{x} \Omega_{c e}(s)}{m_{e} c^{2}}\right)^{-1 / 2} d s}{\int_{0}^{s_{\max }} \omega\left(\gamma^{2}-1-\frac{2 I_{x} \Omega_{c e}(s)}{m_{e} c^{2}}\right)^{-1 / 2} d s}
\end{aligned}
$$
next section, to check the derived map (15), we compare the results provided by this map with results of test particle simulations, as well as with results obtained by solving the full kinetic equation.

\section{VERIFICATION OF MAPPING RESULTS}

Let us fix $h$ and consider a 1D energy distribution $\Psi(\gamma)$ (note that $\Psi$ is a cut of the $2 \mathrm{D}$ energy/pitch-angle dis- 

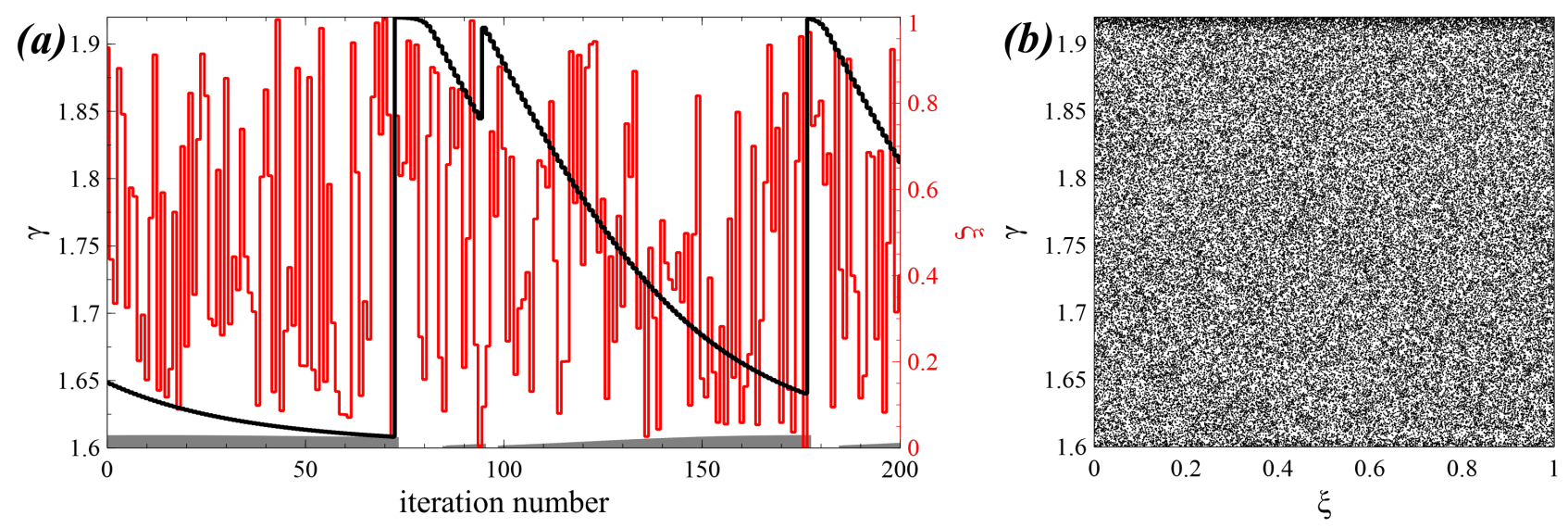

FIG. 4: (a) Particle trajectory for map (15): $\gamma$ and $\xi$ as functions of iteration number, grey color shows the capture probability $\Pi$. (b) Particle trajectory in $(\xi, \gamma)$ plane for $10^{5}$ iterations. $\left(h / m_{e} c^{2}=1.454\right.$ corresponds to, e.g., $\alpha_{0}=45^{\circ}$ of the equatorial pitch-angle for $300 \mathrm{keV}$ electron energy; see details of model parameters in the caption of Fig. 1).

tribution). We can represent this distribution as a set of $10^{6}$ individual particles with different initial energies and randomly distributed phases. Then, the trajectory of each particle can be traced numerically using Hamiltonian equations (1) over a time interval including many resonance interactions. This method reproduces the evolution of $\Psi(\gamma)$ driven by the wave-particle resonant interaction. Alternatively, we can trace trajectories and reproduce the evolution of $\Psi(\gamma)$ using the map (15). The third approach is to solve the kinetic equation that describes $\Psi$ evolution due to nonlinear resonant interactions ${ }^{13,16}$ :

$$
\frac{\partial \Psi}{\partial t}=V \frac{\partial \Psi}{\partial J}+\frac{d V}{d J}\left(\Psi^{*}-\Psi\right) \Theta(J)
$$

where $J(\gamma)=\int^{\gamma} \tau_{b}\left(\gamma^{\prime}\right) d \gamma^{\prime}, V=\Delta \gamma_{s c a t} / \tau_{b}, \Psi^{*}=\Psi\left(\gamma^{*}\right)$ with $\gamma^{*}+\Delta \gamma_{\text {trap }}\left(\gamma^{*}\right)=\gamma$, and $\Theta=0$ for $\Pi=0$ and $\Theta=1$ for $\Pi>0$. Equation (16) can be re-written in a simplified form

$$
\frac{\partial \Psi}{\partial t}=V \frac{\partial \Psi}{\partial \gamma}+\frac{d V}{d \gamma}\left(\Psi^{*} \tau_{b}\left(\gamma^{*}\right) / \tau_{b}(\gamma)-\Psi\right) \Theta(\gamma)+\ell
$$

where the term $\ell$ includes derivatives $\sim \partial \tau_{b} / \partial \gamma$ and can be omitted for a sufficiently weak $\tau_{b}(\gamma)$ dependence (see details of $\gamma \rightarrow J(\gamma)$ transformation in Refs. 14 and 16).

We consider such three types of solutions of $\Psi(\gamma)$ evolution (test particles, Eq. (16), and map (15)) for two initial distributions $\Psi$. Figure 5 shows these three solutions for initial power law energy distribution and three moments of time (note that solutions obtained via test particle simulations and Eq. (16) depend on time, whereas the map (15) depends on the iteration number that should be transformed to time using the bounce period $\tau_{b}(\gamma)$ for each trajectory). All three solutions show a very similar evolution of $\Psi(\gamma)$ : the distribution gets flattened within the resonant energy range (where $\Delta \gamma_{\text {scat }} \neq 0$ ) and forms a plateau. This is the effect of a competition between trapping (energy increase) and scattering (energy decrease), that ultimately results in a uniform distribution (note that this uniform distribution is formed along the resonance curves ${ }^{51,73}$, i.e. for $h=$ const). A similar evolution, although more complicated, can be seen in Fig. 6 showing three solutions for an initial $\Psi(\gamma)$ with a local maximum. This maximum starts drifting to lower energies (due to scattering), whereas a new maximum forms at high energies (due to trapping acceleration). Finally $\Psi(\gamma)$ will be flattened and form a plateau within the resonant energy range. Such an evolution of $\Psi(\gamma)$ has been predicted and described (considering solutions of Eq. (16)) in Refs. 10 and 54.

\section{DISCUSSION}

In this study, we have developed a map describing the dynamics of systems with nonlinear resonant waveparticle interactions. For illustration we used wave and background plasma parameters typical for the Earth inner magnetosphere, where relativistic electrons resonate with high amplitude whistlers. This system is well investigated in the regime of low wave amplitudes where quasi-linear diffusion equations are applicable ${ }^{51,69,78}$, but so far there is no method allowing to model the long-term evolution of this system in the presence of nonlinear resonant effects. One of the most widespread technique, test particle simulation ${ }^{4,23,26,27,37,53,62}$, provides a lot of important information about electron acceleration and scattering rates, but such simulations are limited to quite short time intervals. This limitation mostly comes from the need to integrate the entire (bounce) particle trajectory even if energy and magnetic moment only change at the locations of wave-particle resonances. Therefore, a natural solution consist in considering only resonanceinduced changes of particle energy and pitch-angle, like in the quasi-linear diffusion approach. The generalization 

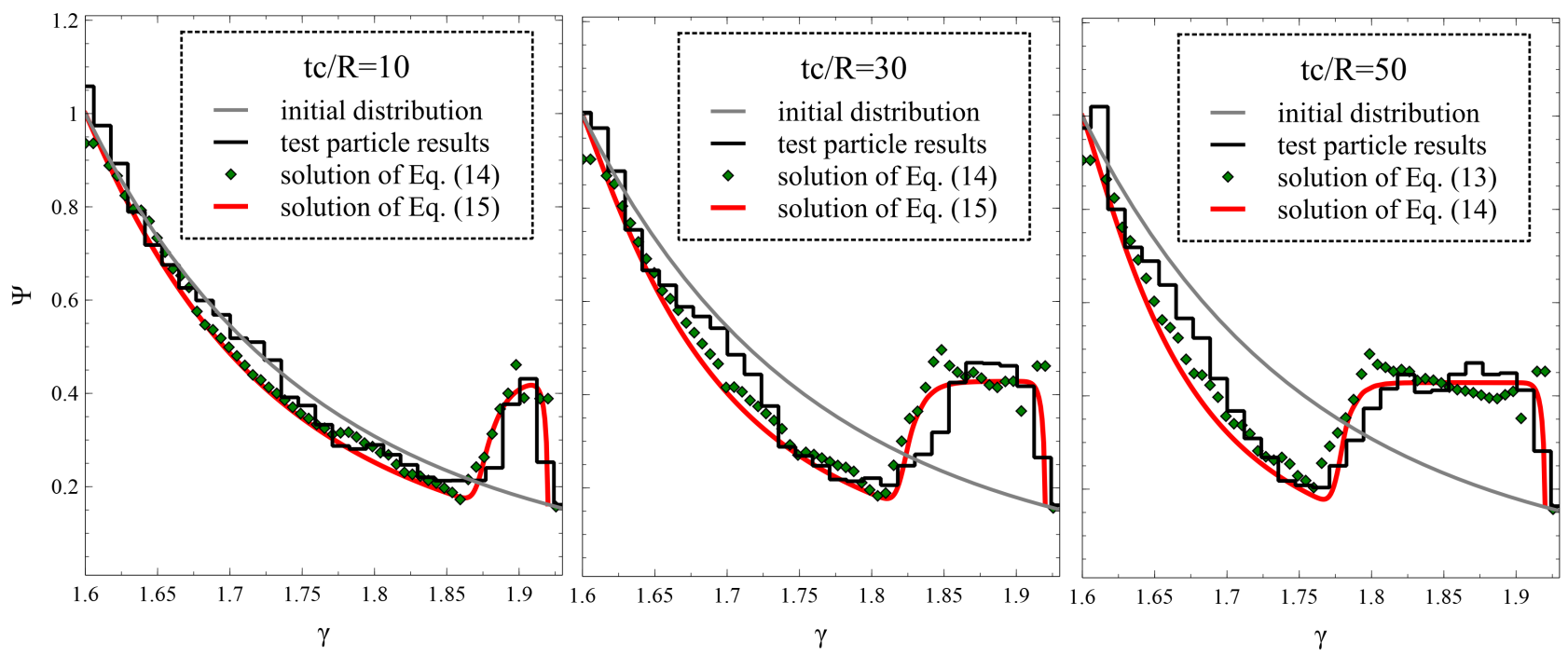

FIG. 5: Evolution of distribution $\Psi(\gamma)$ for $h / m_{e} c^{2}=1.454$ (this value of $h$ corresponds to, e.g., equatorial pitch-angle $\alpha_{0}=45^{\circ}$ for $300 \mathrm{keV}$ electron energy; see details of the model parameters in the caption to Fig. 1): black color shows results of test particle simulations ( $10^{6}$ trajectories), red color shows solutions of Eq. (16), green color shows results of mapping (15). The initial distribution $\Psi(\gamma)$ is shown in all panels with grey curves. Time is normalized on $R / c$ (a scale of the quarter of the bounce period) with $R=4.5$ Earth radii.
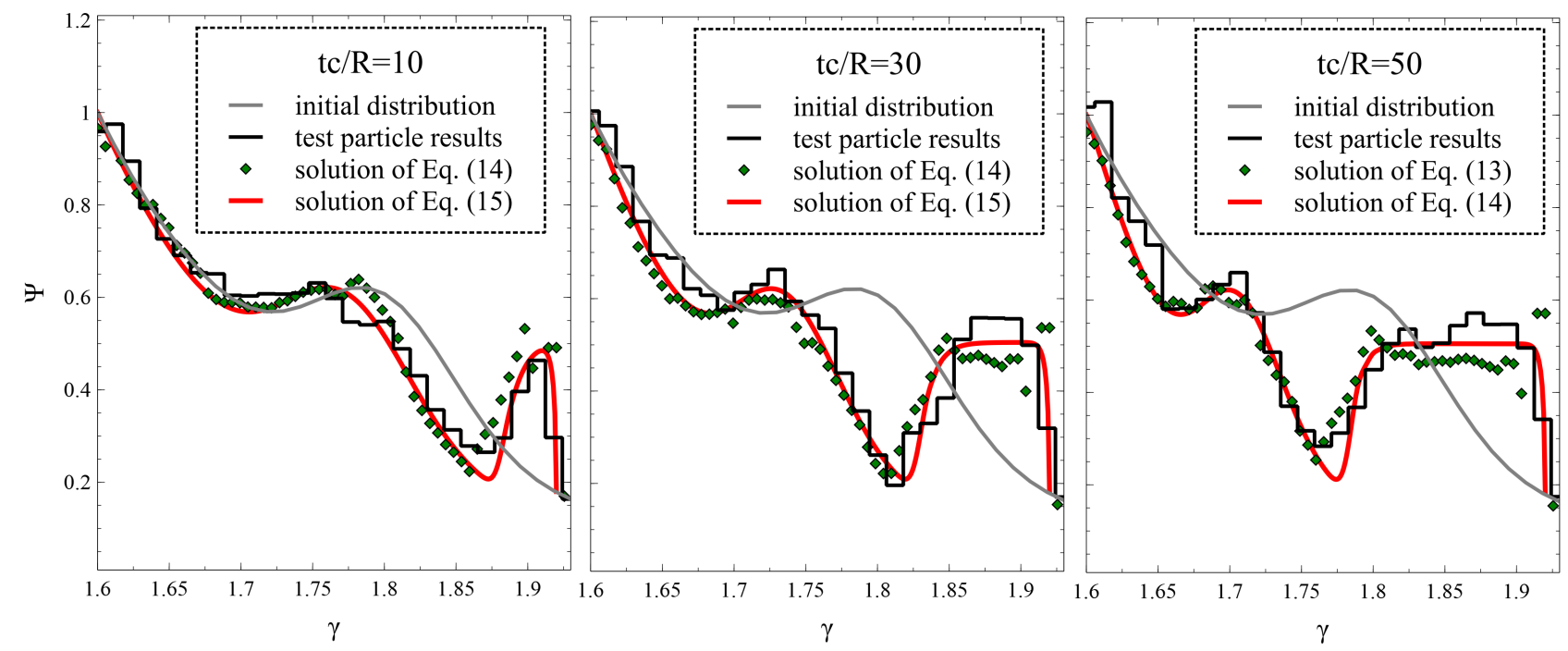

FIG. 6: Evolution of the distribution $\Psi(\gamma)$ for $h / m_{e} c^{2}=1.454$ (this value of $h$ corresponds to, e.g., equatorial pitch-angle $\alpha_{0}=45^{\circ}$ for $300 \mathrm{keV}$ electron energy; see details of the model parameters in the caption of Fig. 1): black color shows results of test particle simulations $\left(10^{6}\right.$ trajectories), red color shows solutions of Eq. (16), green color shows results of mapping (15). The initial distribution $\Psi(\gamma)$ is shown in all panels with grey curves. Time is normalized to $R / c$ (the quarter of the bounce period) with $R=4.5$ Earth radii.

of the diffusion equation with inclusion of terms related to nonlinear wave-particle interaction results in Eq. (16) or similar types of kinetic equations ${ }^{13,16,70}$. However, this kinetic equation still relies on the assumption of a uniform distribution of resonant phases (i.e., it excludes effects related to phase correlation at multiple passages through the resonance) and it cannot be easily generalized for systems with multiple waves. These two prob- lems can be resolved using the map approach that includes resonant phase dynamics while also allowing the inclusion of many resonances. Let us consider these two issues in more details.

Assuming a uniform distribution of resonant phases corresponds to the assumption that two successive resonances are not correlated, i.e., that electron energy jumps $\Delta \gamma$ (due to trapping or scattering) can be considered as 
independent over a long run. This important property of the resonant system usually results from the dependence of the phase gain $\Delta \zeta$ on energy $\gamma$ (see Eq. (15)). This gain is usually large $\Delta \zeta \sim \omega \tau_{b} \gg 1$ (since whistler wave period is much smaller than the electron bounce period along magnetic field lines) and, thus, even a small change of energy $\Delta \gamma$ due to resonant interaction should result in a significant change of $\Delta \zeta: \delta(\Delta \zeta) \sim(\partial \Delta \zeta / \partial \gamma) \Delta \gamma$, justifying the assumption of randomly distributed phases. However, resonances can be correlated (and the distribution of resonant phases can be non-uniform, see Ref. 12) for systems with small $\partial \Delta \zeta / \partial \gamma$. Such a situation can hardly appear in the Earth radiation belts, but it is more realistic for resonant electron interaction with strong electrostatic waves and solitons around the bow shock $^{88}$. This corresponds to Landau resonant interaction without the term $\varpi / \gamma$ in Eq. (15) and with the time interval between resonances $\sim \tau_{b}$ weakly depending on energy. Therefore, the proposed map technique may be useful for investigations of nonlinear wave-particle interactions in such systems, where the assumption of a uniform distribution of resonant phases is not applicable.

The map (15) has been constructed for a system with a single wave (single resonance). In this system the condition $h=$ const reduces the initially 2D space (energy/pitch-angle or $\left(\gamma, I_{x}\right)$ ) to $1 \mathrm{D}$ space. However, unlike kinetic equation (16), this map can be generalized to many resonances resulting in particle motion in the $\left(\gamma, I_{x}\right)$ space. Indeed, the map describes change of the resonant phase $\zeta$ between two resonances and energy change on the resonance. The $\zeta$ change can be modified by replacing the integration over the entire bounce period with the integration between two resonances in Eq. (14), whereas the energy change can be replaced with energy and $I_{x}$ changes. This generalization looks much simpler to achieve than the corresponding generalization of the kinetic equation (16).

Figure 4 shows that after many iterations the particle trajectory fills the entire available space in the $(\zeta, \gamma)$ space. For ensembles including many trajectories, the final state of the distribution function will be a uniform distribution where phase space density $\Psi$ should have the same value for all energies. This is the final state for both quasi-linear diffusion, that tends to reduce gradients of $\Psi$ along the resonance curve, and nonlinear wave-particle interaction described by Eq. (16), which has only one stationary solution $\Psi=$ const (see Ref. 10). Therefore, the map (15) describes distribution flattening, $\partial \Psi / \partial \gamma \rightarrow 0$, and allows to estimate a typical timescale of this flattening. For example, Fig. 7 shows the evolution of the dispersion $D=\sqrt{\left\langle\gamma^{2}\right\rangle-\langle\gamma\rangle^{2}}$ of distribution $\Psi$ for four different initial $\Psi$ (shown in the inserted panel). The dynamics of $\Psi$ is described by $10^{5}$ trajectories of map (15), and $D$ is normalized to the dispersion of the uniform distribution for the same $\gamma$ range (i.e., $D / D_{0} \approx 1$ means $\Psi \approx$ const). As the map (15) describes discrete changes of energy with time, the beginning of the $D$ evolution

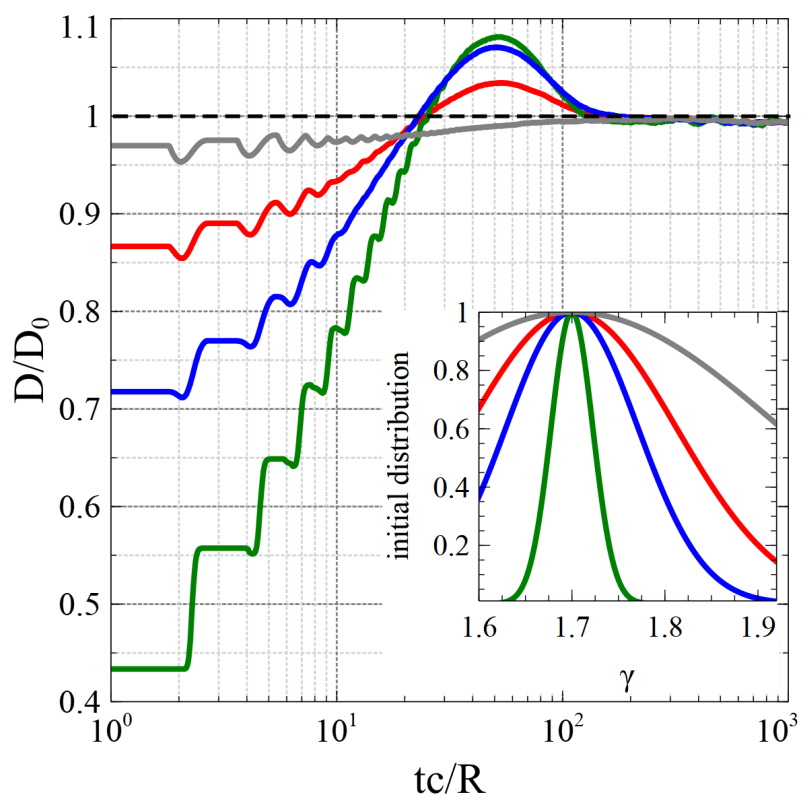

FIG. 7: Evolution of dispersion $D=\sqrt{\left\langle\gamma^{2}\right\rangle-\langle\gamma\rangle^{2}}$ for four distributions $\Psi(\gamma)$ and $h / m_{e} c^{2}=1.454$ (this value of $h$ corresponds to, e.g. $45^{\circ}$ of the equatorial pitch-angle for $300 \mathrm{keV}$ electron energy; see details of model parameters in the caption of Fig. 1). Dispersions are normalized to the dispersion $D_{0}$ of the uniform distribution $\Psi=$ const.

consists of step-like jumps (note that we transformed the iteration number to time for each trajectory to plot $D$ versus time). Independently of the initial distribution, $D / D_{0} \approx 1$ after $\approx 100 \tau_{b}$, and this timescale is much shorter than typical quasi-linear time scales ${ }^{48,79}$.

To conclude, in this paper we have considered nonlinear resonances between relativistic electrons and intense whistler-mode waves. We have demonstrated that the long-term dynamics of the electron distribution can be described by a map taking into account the important interdependence between the probability of trapping $\Pi$ and energy change due to scattering $\Delta \gamma_{\text {scat }}$ : $\Pi=-d \Delta \gamma_{s c a t} / d \gamma$. This map is different from the classical Chirikov map ${ }^{25}$, and allows to describe both effects of phase trapping and nonlinear scattering. The proposed mapping technique can be useful for the description of charged particle acceleration in various space plasma systems including the Earth radiation belts and the Earth bow shock.

\section{ACKNOWLEDGMENTS}

Authors are grateful to Dr. D. Mourenas for useful discussion. This work was supported by the Russian Scientific Foundation, project 19-12-00313. 


\section{APPENDIX A}

In this Appendix we derive formula (12) which expresses variation of variable $\xi$ between two resonance crossings via gain of phase $\zeta$ between these crossings. We consider quite general form of Hamiltonian. Result of our derivation is directly applicable to the particular system considered in the main text. This derivation is somewhat similar to the derivation provided in Ref. 58 for change of phase between separatrix crossings. Important to notice that, following the system description in the main text, we consider one resonance crossing per one period of slow motion, i.e. the two resonance crossings are separated in time by one slow period.

Let us consider a general Hamiltonian as the sum of an unperturbed part $H_{0}(I, p, q)$ and a small perturbation $\varepsilon H_{1}(I, \zeta, p, q)$ (with $\varepsilon \ll 1$; note in the main text $\left.\varepsilon \sim B_{w} / B_{0}\right)$ where $\left(q, \varepsilon^{-1} p\right),(\zeta, I)$ are pairs of conjugate variables (hence $(\zeta, I)$ are fast variables, $(q, p)$ are slow variables), and $H_{1}$ is periodic in $\zeta$. Momentum $I$ is an adiabatic invariant: $\dot{I}=-\varepsilon \partial H_{1} / \partial \zeta$, and $I$ is constant in the averaged over $\zeta$ system. There is no explicit dependence on time, and thus $H=h=$ const. The resonance condition is determined by the equation $\partial H_{0} / \partial I=0$. Solving this equation for $I$ gives the equation $I=I_{R}(p, q)$ of the resonance surface. Denote $\Lambda(p, q)=H_{0}\left(I_{R}(p, q), p, q\right)$. The Hamiltonian can be expanded around the resonance surface similarly to Eq. (5). We assume that the phase portrait of the expanded Hamiltonian looks like one shown in Fig. 8.

We introduce the improved adiabatic invariant $J$ with the variable transformation $(I, \zeta, p, q) \mapsto(J, \theta, P, Q)$ such that the new Hamiltonian is $H=H_{0}(J, P, Q)+$ $\varepsilon \bar{H}_{1}(J, P, Q)$ where $\bar{H}_{1}$ is the average of $H_{1}$ over $\zeta$ (in the leading approximation).

Far from the resonance $\theta$ changes with the frequency

$$
\dot{\theta}=\frac{\partial H_{0}}{\partial J}+\varepsilon \frac{\partial \bar{H}_{1}}{\partial J}
$$

with $J=$ const, and

$$
\dot{Q}=\varepsilon \frac{\partial H_{0}}{\partial P}+\varepsilon^{2} \frac{\partial \bar{H}_{1}}{\partial P}, \quad \dot{P}=-\varepsilon \frac{\partial H_{0}}{\partial Q}-\varepsilon^{2} \frac{\partial \bar{H}_{1}}{\partial Q}
$$

We introduce $\omega_{0}(J, P, Q)=\partial H_{0} / \partial J, \omega_{1}(J, P, Q)=$ $\partial \bar{H}_{1} / \partial J$, and consider large number $N \gg 1$ of rounds of $\zeta$ from $t=t_{0}$ (when phase point is far from the resonance and moves towards the resonance) to $t=t_{N}$; the last round is sufficiently far from the resonance and $\theta \approx \zeta$ in the leading approximation. The last round ends at $\zeta=\zeta_{c} \bmod 2 \pi$ (see Fig. 8 for the definition of $\zeta_{c}$ ). Then

$$
\zeta_{c N}=\zeta_{0}+\int_{t_{0}}^{t_{N}}\left(\omega_{0}+\varepsilon \omega_{1}\right) d t \bmod 2 \pi
$$

where $\zeta_{c N}$ is $\zeta_{c}$ value at $t=t_{N}$. We introduce $t_{*}$ as the time of crossing the resonance, i.e. $\left.\omega_{0}(J, P, Q)\right|_{t=t_{*}}=0$,

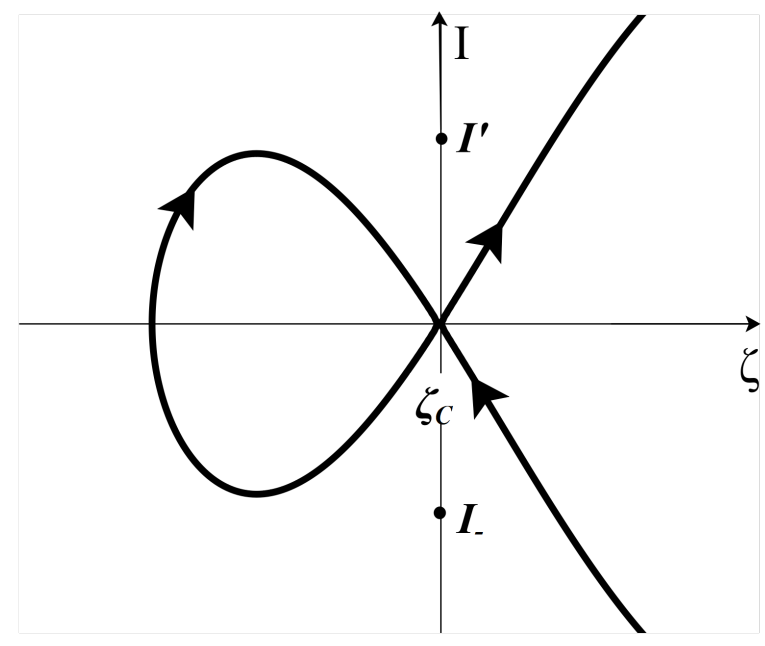

FIG. 8: Schematic of phase portrait.

and rewrite Eq. (20):

$\zeta_{c N}=\zeta_{0}+\int_{t_{0}}^{t_{*}}\left(\omega_{0}+\varepsilon \omega_{1}\right) d t-\int_{t_{N}}^{t_{*}}\left(\omega_{0}+\varepsilon \omega_{1}\right) d t \bmod 2 \pi$

Because $t_{*}-t_{N} \ll 1 / \varepsilon$ we can use $\dot{Q}=\varepsilon \partial H_{0} / \partial P, \dot{P}=$ $-\varepsilon \partial H_{0} / \partial P$ and $Q \approx q, P \approx p$ in the last integral in Eq. (21). We also assume that $\zeta_{c N} \approx \zeta_{c *}=\left.\zeta_{c}\right|_{t=t_{*}}$. To describe system dynamics for $t \in\left[t_{N}, t_{*}\right]$ we use the expansion of the Hamiltonian around the resonance: $H=$ $\Lambda+F$ and

$$
F=\frac{1}{2} g\left(I-I_{R}\right)^{2}+\varepsilon H_{1}, \quad g=\left.\frac{\partial^{2} H_{0}}{\partial I^{2}}\right|_{I=I_{R}} \approx \text { const }
$$

The Hamiltonian in new variables $(J, \theta, P, Q)$ can be expanded as

$$
H=\Lambda+\frac{1}{2} g\left(J-I_{R}\right)^{2}+\varepsilon \bar{H}_{1}
$$

We introduce $e=g\left(J-I_{R}\right)^{2} / 2$ and write

$$
\begin{aligned}
\dot{e} & =\varepsilon g\left(J-I_{R}\right) r, \quad r=-\left\{I_{R}, \Lambda\right\} \approx \text { const } \\
\omega_{0} & =\frac{\partial H}{\partial J}=g\left(J-I_{R}\right)
\end{aligned}
$$

Using $d t=d e /(d e / d t)$ we rewrite integral

$$
\int_{t_{N}}^{t_{*}}\left(\omega_{0}+\varepsilon \omega_{1}\right) d t \approx \frac{1}{\varepsilon} \int_{e_{N}}^{0} \frac{\omega_{0} d e}{g\left(J-I_{R}\right) r}=\frac{1}{\varepsilon} \int_{e_{N}}^{0} \frac{d e}{r}=-\frac{e_{N}}{\varepsilon r}
$$

where $e_{N}$ is the value $e$ along the trajectory at $t=t_{N}$, and we omit $\varepsilon \omega_{1}$ because $t_{*}-t_{N} \ll 1 / \varepsilon$. Using $e+\varepsilon \bar{H}_{1}=$ $F$, we write

$$
e_{N}=F_{N}-\varepsilon \bar{H}_{1} \approx F_{N}-\varepsilon \bar{H}_{1 *}
$$


where $F_{N}$ is the value $F$ along the trajectory at $t=t_{N}$, and $\bar{H}_{1 *}$ is the resonant value of $\bar{H}_{1}$. Substituting Eqs. $(25,26)$ to Eq. (21), we obtain

$$
\zeta_{c *}=\zeta_{0}+\int_{t_{0}}^{t_{*}}\left(\omega_{0}+\varepsilon \omega_{1}\right) d t+\frac{F_{N}-\varepsilon \bar{H}_{1 *}}{\varepsilon r} \bmod 2 \pi
$$

or

$$
\frac{F_{N}}{2 \pi \varepsilon r}=\frac{\bar{H}_{1 *}}{2 \pi r}-\frac{1}{2 \pi}\left(\zeta_{0}-\zeta_{c *}+\int_{t_{0}}^{t_{*}}\left(\omega_{0}+\varepsilon \omega_{1}\right) d t\right) \bmod 1
$$

We define $F_{\text {last }}$ as the value of $F$ along the trajectory at the last crossing of the line $\zeta=\zeta_{c}$ before crossing the resonance. Thus, $F_{l a s t}=F_{N} \bmod 2 \pi \varepsilon r$, because the change of $F$ for one round of $\zeta$ equals $2 \pi \varepsilon r$. We introduce $\xi=\left(F_{\text {last }}-\varepsilon H_{1 c *}\right) /(2 \pi \varepsilon r)$, where $H_{1 c *}$ is value of $H_{1}$ at $\zeta=\zeta_{c}, t=t_{*}$ and write

$\xi=\operatorname{Frac}\left(\frac{\bar{H}_{1 *}-H_{1 c *}}{2 \pi r}-\frac{\zeta_{0}-\zeta_{c *}}{2 \pi}-\frac{1}{2 \pi \varepsilon} \int_{\tau_{0}}^{\tau_{*}}\left(\omega_{0}+\varepsilon \omega_{1}\right) d \tau\right)$

Here $\tau=\varepsilon t, \tau_{*}=\varepsilon t_{*}$. Note that $\xi$ can be written as

$$
\begin{aligned}
\xi & =\frac{F_{\text {last }}-\varepsilon H_{1 c *}}{2 \pi \varepsilon r}=\frac{F_{\text {last }}-\varepsilon r \zeta_{c *}-\left(\varepsilon H_{1 c *}-\varepsilon r \zeta_{c *}\right)}{2 \pi \varepsilon r} \\
& =\frac{E_{\text {last }}-E_{c *}}{2 \pi \varepsilon r}
\end{aligned}
$$

where

$$
E=\frac{1}{2} g\left(I-I_{R}\right)^{2}-\varepsilon r \zeta+\varepsilon H_{1},
$$

and $E_{c *}$ is value of $E$ at $\zeta=\zeta_{c}, t=t_{*}, E_{\text {last }}$ is the value of $E$ at the last crossing of the line $\zeta=\zeta_{c}$ before crossing the resonance.

Let us use Eq. (29) to consider two successive resonance crossings (note there is only one resonant interaction for one period of slow motion, i.e. two successive resonance crossings are separated by one slow period). Far from the resonance the improved adiabatic invariant $J$ can be considered as a constant. Denote $\tau_{-}$and $\tau_{+}$ slow time moments of the resonance crossings $(\tau=\varepsilon t)$. Let $\xi_{ \pm}$be values of the variable $\xi$ corresponding to these two crossings. We are looking for a relation between $\xi_{+}$ and $\xi_{-}$. Due to periodicity of the slow motion, values of $\zeta_{c *}, H_{1 c *}, \bar{H}_{1 *}, r$ are the same at $\tau=\tau_{+}$and $\tau=\tau_{-}$.

We consider value $\xi_{-}$for the first of the resonance crossings, and the corresponding value $E=E_{\text {last-. }}$ At $\tau=\tau_{-}$the phase point is on the line $\zeta=\zeta_{c *}$ with $I<I_{R}$ at the position indicated by the symbol $I_{-}$in Fig. 8 . We assume that this phase point crosses the resonance without trapping. Thus, at some $\tau=\tau^{\prime}$ it arrives again to the line $\zeta=\zeta_{c}=\zeta_{c *}^{\prime}$ with the value $E=E^{\prime}$ and $I>I_{R}$. The phase point position is indicated by the symbol $I^{\prime}$ in Fig. 8. We denote $\xi^{\prime}=\left(E^{\prime}-E_{c *}\right) /(2 \pi \varepsilon r)$. We have $E^{\prime} \approx E_{\text {last- }}, \xi^{\prime} \approx \xi_{-}$.
At some moment of the slow time $\tau_{0} \in\left(\tau_{-}, \tau_{+}\right)$the phase point is far from the resonance and has $\zeta=\zeta_{0}$. Then Eq. (29) gives

$\xi_{+}=\operatorname{Frac}\left(\frac{\bar{H}_{1 *}-H_{1 c *}}{2 \pi r}-\frac{\zeta_{0}-\zeta_{c *}}{2 \pi}-\frac{1}{2 \pi \varepsilon} \int_{\tau_{0}}^{\tau_{+}}\left(\omega_{0}+\varepsilon \omega_{1}\right) d \tau\right)$

Similarly, considering the backward motion on the time interval from $\tau_{0}$ to $\tau^{\prime}$ we get

$\xi^{\prime}=\operatorname{Frac}\left(\frac{\bar{H}_{1 *}-H_{1 c *}}{2 \pi r}-\frac{\zeta_{0}-\zeta_{c *}}{2 \pi}+\frac{1}{2 \pi \varepsilon} \int_{\tau^{\prime}}^{\tau_{0}}\left(\omega_{0}+\varepsilon \omega_{1}\right) d \tau\right)$

In this expression we can replace in the leading approximation $\xi^{\prime}$ with $\xi_{-}$and $\tau^{\prime}$ with $\tau_{-}$(note that

$$
\frac{1}{2 \pi \varepsilon} \int_{\tau_{-}}^{\tau^{\prime}}\left(\omega_{0}+\varepsilon \omega_{1}\right) d \tau
$$

is small, because $\omega_{0}$ vanishes on the resonant surface). Thus for the value $\Delta \xi=\xi_{+}-\xi_{-}$we get

$$
\Delta \xi=\xi_{+}-\xi_{-}=-\frac{1}{2 \pi \varepsilon} \int_{\tau_{-}}^{\tau_{+}}\left(\omega_{0}+\varepsilon \omega_{1}\right) d \tau \bmod 1
$$

and this is the phase $\zeta$ gain between two resonance crossings (between moments $\tau_{-}$and $\tau_{+}$) normalized on $2 \pi$ and taken with the minus sign. In the main text $\bar{H}_{1}=0$ and thus $\omega_{1}=\partial \bar{H}_{1} / \partial J=0$.

${ }^{1}$ Agapitov, O., Artemyev, A., Krasnoselskikh, V., Khotyaintsev, Y.V., Mourenas, D., Breuillard, H., Balikhin, M., Rolland, G., 2013. J. Geophys. Res. 118, 3407-3420. doi:10.1002/jgra.50312.

${ }^{2}$ Agapitov, O., Artemyev, A., Mourenas, D., Krasnoselskikh, V., Bonnell, J., Le Contel, O., Cully, C.M., Angelopoulos, V., 2014. J. Geophys. Res. 119, 1606-1626. doi:10.1002/2013JA019223.

${ }^{3}$ Agapitov, O.V., Artemyev, A.V., Mourenas, D., Mozer, F.S., Krasnoselskikh, V., 2015. Geophys. Res. Lett. 42, 10. doi: 10.1002/2015GL066887.

${ }^{4}$ Agapitov, O.V., Mourenas, D., Artemyev, A.V., Mozer, F.S., 2016. Geophys. Res. Lett. 43, 11,112-11,120. URL: http://dx. doi.org/10.1002/2016GL071250, doi:10.1002/2016GL071250.

${ }^{5}$ Albert, J.M., 1993. Physics of Fluids B 5, 2744-2750. doi: $10.1063 / 1.860715$.

${ }^{6}$ Albert, J.M., 2002. Geophys. Res. Lett. 29, 1275 . doi: 10.1029/2001GL013941.

${ }^{7}$ Albert, J.M., Tao, X., Bortnik, J., 2013. Aspects of Nonlinear Wave-Particle Interactions, in: Summers, D., Mann, I.U., Baker, D.N., Schulz, M. (Eds.), Dynamics of the Earth's Radiation Belts and Inner Magnetosphere. doi: 10.1029/2012GM001324.

${ }^{8}$ An, X., Van Compernolle, B., Bortnik, J., Thorne, R.M., Chen, L., Li, W., 2016. Geophys. Res. Lett. 43, 2413-2421. doi: 10.1002/2015GL067126.

${ }^{9}$ Artemyev, A., Agapitov, O., Mourenas, D., Krasnoselskikh, V., Shastun, V., Mozer, F., 2016. Space Science Reviews 200, 261355. URL: http://dx.doi.org/10.1007/s11214-016-0252-5, doi:10.1007/s11214-016-0252-5. 
${ }^{10}$ Artemyev, A., Neishtadt, A., Vasiliev, A., 2019. Physica D: Nonlinear Phenomena URL: http://www.sciencedirect. com/science/article/pii/S0167278918304433, doi: https://doi.org/10.1016/j.physd.2018.12.007.

${ }^{11}$ Artemyev, A.V., Neishtadt, A.I., Vainchtein, D.L., Vasiliev, A.A., Vasko, I.Y., Zelenyi, L.M., 2018a. Communications in Nonlinear Science and Numerical Simulations 65, 111-160. doi: 10.1016/j.cnsns.2018.05.004.

${ }^{12}$ Artemyev, A.V., Neishtadt, A.I., Vasiliev, A.A., 2019. arXiv e-prints, arXiv:1904.02973arXiv: 1904.02973.

${ }^{13}$ Artemyev, A.V., Neishtadt, A.I., Vasiliev, A.A., Mourenas, D., 2016. Physics of Plasmas 23, 090701. doi:10.1063/1.4962526.

${ }^{14}$ Artemyev, A.V., Neishtadt, A.I., Vasiliev, A.A., Mourenas, D., 2017a. ArXiv e-prints arXiv: 1710.04489

${ }^{15}$ Artemyev, A.V., Neishtadt, A.I., Vasiliev, A.A., Mourenas, D., 2017b. Phys. Rev. E 95, 023204. doi: 10.1103/PhysRevE.95.023204.

${ }^{16}$ Artemyev, A.V., Neishtadt, A.I., Vasiliev, A.A., Mourenas, D., 2018b. Journal of Plasma Physics 84, 905840206. doi: $10.1017 /$ S0022377818000260.

${ }^{17}$ Artemyev, A.V., Neishtadt, A.I., Zelenyi, L.M., 2011. Chaos 21, 043120. doi:10.1063/1.3657916.

${ }^{18}$ Artemyev, A.V., Vasiliev, A.A., Mourenas, D., Agapitov, O., Krasnoselskikh, V., Boscher, D., Rolland, G., 2014a. Geophys. Res. Lett. 41, 5727-5733. doi:10.1002/2014GL061380.

${ }^{19}$ Artemyev, A.V., Vasiliev, A.A., Mourenas, D., Agapitov, O.V., Krasnoselskikh, V.V., 2014b. Physics of Plasmas 21, 102903. doi:10.1063/1.4897945.

${ }^{20}$ Artemyev, A.V., Vasiliev, A.A., Mourenas, D., Neishtadt, A.I., Agapitov, O.V., Krasnoselskikh, V., 2015. Physics of Plasmas 22, 112903. doi:10.1063/1.4935842.

${ }^{21}$ Bell, T.F., 1984 . J. Geophys. Res. 89, 905-918. doi: 10.1029/JA089iA02p00905.

${ }^{22}$ Benkadda, S., Sen, A., Shklyar, D. R., 1996. Chaos 6, 451-460. doi:10.1063/1.166187.

${ }^{23}$ Bortnik, J., Thorne, R.M., Inan, U.S., 2008. Geophys. Res. Lett. 35, 21102. doi:10.1029/2008GL035500.

${ }^{24}$ Cattell, C., Wygant, J.R., Goetz, K., Kersten, K., Kellogg, P.J., von Rosenvinge, T., Bale, S.D., Roth, I., Temerin, M., Hudson, M.K., Mewaldt, R.A., Wiedenbeck, M., Maksimovic, M., Ergun, R., Acuna, M., Russell, C.T., 2008. Geophys. Res. Lett. 35, 1105. doi:10.1029/2007GL032009.

${ }^{25}$ Chirikov, B.V., 1979. Physics Reports 52, 263-379. doi: 10.1016/0370-1573(79)90023-1.

${ }^{26}$ Demekhov, A.G., Trakhtengerts, V.Y., Rycroft, M., Nunn, D., 2009. Geomagnetism and Aeronomy 49, 24-29. doi: 10.1134/S0016793209010034.

${ }^{27}$ Demekhov, A.G., Trakhtengerts, V.Y., Rycroft, M.J., Nunn, D., 2006. Geomagnetism and Aeronomy 46, 711-716. doi: 10.1134/S0016793206060053.

${ }^{28} \mathrm{Fu}$, X., Cowee, M.M., Friedel, R.H., Funsten, H.O., Gary, S.P., Hospodarsky, G.B., Kletzing, C., Kurth, W., Larsen, B.A., Liu, K., MacDonald, E.A., Min, K., Reeves, G.D., Skoug, R.M., Winske, D., 2014. Journal of Geophysical Research (Space Physics) 119, 8288-8298. doi:10.1002/2014JA020364.

${ }^{29}$ Furuya, N., Omura, Y., Summers, D., 2008. J. Geophys. Res. 113, 4224. doi:10.1029/2007JA012478.

${ }^{30}$ Gary, S.P., Wang, J., 1996. J. Geophys. Res. 101, 10749-10754. doi:10.1029/96JA00323.

${ }^{31}$ Hsieh, Y.K., Omura, Y., 2017. J. Geophys. Res. 122, 675-694. doi:10.1002/2016JA023255.

${ }^{32}$ Hsieh, Y.K., Omura, Y., 2017. Radio Science 52, 1268 1281. URL: http://dx.doi.org/10.1002/2017RS006245, doi: 10.1002/2017RS006245. 2017RS006245.

${ }^{33}$ Itin, A.P., Neishtadt, A.I., Vasiliev, A.A., 2000. Physica D: Nonlinear Phenomena 141, 281-296. doi:10.1016/S01672789(00)00039-7.

${ }^{34}$ Karpman, V.I., 1974. Space Sci. Rev. 16, 361-388. doi: $10.1007 / \mathrm{BF} 00171564$

${ }^{35}$ Karpman, V.I., Istomin, J.N., Shklyar, D.R., 1974. Plasma
Physics 16, 685-703. doi:10.1088/0032-1028/16/8/001.

${ }^{36}$ Katoh, Y., Omura, Y., 2007a. Geophys. Res. Lett. 34, 3102. doi:10.1029/2006GL028594.

${ }^{37}$ Katoh, Y., Omura, Y., 2007b. Geophys. Res. Lett. 34, L13102. doi:10.1029/2007GL029758.

${ }^{38}$ Kennel, C.F., Engelmann, F., 1966. Physics of Fluids 9, 23772388. doi:10.1063/1.1761629.

${ }^{39}$ Kennel, C.F., Wong, H.V., 1967. Journal of Plasma Physics 1, 75. doi:10.1017/S002237780000310X.

${ }^{40}$ Khazanov, G.V., Tel'nikhin, A.A., Kronberg, T.K., 2013. Journal of Geophysical Research (Space Physics) 118, 6397-6404. doi:10.1002/2013JA019122.

${ }^{41}$ Khazanov, G.V., Tel'nikhin, A.A., Kronberg, T.K., 2014. Nonlinear Processes in Geophysics 21, 61-85. doi:10.5194/npg-2161-2014.

${ }^{42}$ Kitahara, M., Katoh, Y., 2019. J. Geophys. Res. 124, 55685583. doi:10.1029/2019JA026493.

${ }^{43}$ Krafft, C., Volokitin, A.S., Flé, M., 2000. Physics of Plasmas 7, 4423-4432. doi:10.1063/1.1308565.

${ }^{44}$ Kuzichev, I.V., Soto-Chavez, A.R., Park, J., Gerrard, A., Spitkovsky, A., 2019a. Physics of Plasmas 26, 072901. doi: $10.1063 / 1.5096537$.

${ }^{45}$ Kuzichev, I.V., Vasko, I.Y., Rualdo Soto-Chavez, A., Tong, Y., Artemyev, A.V., Bale, S.D., Spitkovsky, A., 2019b. Astrophys. J. 882, 81. doi:10.3847/1538-4357/ab3290, arXiv: 1907.04878.

${ }^{46}$ Lerche, I., 1968 Physics of Fluids 11.

${ }^{47} \mathrm{Li}$, W., Bortnik, J., Thorne, R.M., Angelopoulos, V., 2011. J. Geophys. Res. 116, 12205. doi:10.1029/2011JA017035.

${ }^{48} \mathrm{Li}$, W., Bortnik, J., Thorne, R.M., Cully, C.M., Chen, L., Angelopoulos, V., Nishimura, Y., Tao, J.B., Bonnell, J.W., Lecontel, O., 2013. J. Geophys. Res. 118, 1461-1471. doi: $10.1002 /$ jgra.50176.

${ }^{49} \mathrm{Li}$, W., Mourenas, D., Artemyev, A.V., Bortnik, J., Thorne, R.M., Kletzing, C.A., Kurth, W.S., Hospodarsky, G.B., Reeves, G.D., Funsten, H.O., Spence, H.E., 2016. Geophys. Res. Lett. 43, 8867-8875. doi:10.1002/2016GL070386.

${ }^{50}$ Lichtenberg, A.J., Lieberman, M.A., 1983. Regular and stochastic motion.

${ }^{51}$ Lyons, L.R., Williams, D.J., 1984. Quantitative aspects of magnetospheric physics.

52 Mourenas, D., Artemyev, A.V., Agapitov, O.V., Krasnoselskikh, V., Mozer, F.S., 2015. J. Geophys. Res. 120, 3665-3683. doi: 10.1002/2015JA021135.

${ }^{53}$ Mourenas, D., Artemyev, A.V., Agapitov, O.V., Mozer, F.S., Krasnoselskikh, V.V., 2016. J. Geophys. Res. 121, 4498-4517. doi:10.1002/2015JA022223.

${ }^{54}$ Mourenas, D., Zhang, X.J., Artemyev, A.V., Angelopoulos, V., Thorne, R.M., Bortnik, J., Neishtadt, A.I., Vasiliev, A.A., 2018. J. Geophys. Res. 123, 4979-4999. doi:10.1029/2018JA025417.

${ }^{55}$ Neishtadt, A., 1975. Journal of Applied Mathematics and Mechanics 39, 594-605. doi:10.1016/0021-8928(75)90060-X.

${ }^{56}$ Neishtadt, A.I., 1999. On Adiabatic Invariance in TwoFrequency Systems. in Hamiltonian Systems with Three or More Degrees of Freedom, ed. Simo C., NATO ASI Series C. Dordrecht: Kluwer Acad. Publ. 533, 193-213. doi:10.1063/1.166236.

${ }^{57}$ Neishtadt, A.I., 2014. Russian Mathematical Surveys 69, 771. URL: http://stacks.iop.org/0036-0279/69/i=5/a=771.

${ }^{58}$ Neishtadt, A.I., Vasiliev, A.A, 2005. Nonlinearity 18, 1393. URL: http://stacks.iop.org/Non/18/1393.

${ }^{59} \mathrm{Ni}$, B., Thorne, R.M., Zhang, X., Bortnik, J., Pu, Z., Xie, L., Hu, Z.j., Han, D., Shi, R., Zhou, C., Gu, X., 2016. Origins of the Earth's Diffuse Auroral Precipitation. Space Sci. Rev. 200, 205-259. doi:10.1007/s11214-016-0234-7.

${ }^{60}$ Nunn, D., 1971. Journal of Plasma Physics 6, 291. doi: 10.1017/S0022377800006061.

${ }^{61}$ Nunn, D., Omura, Y., 2015. J. Geophys. Res. 120, 2890-2911. doi:10.1002/2014JA020898.

${ }^{62}$ Omura, Y., Furuya, N., Summers, D., 2007. J. Geophys. Res. 112, 6236. doi:10.1029/2006JA012243.

${ }^{63}$ Omura, Y., Matsumoto, H., Nunn, D., Rycroft, M.J., 1991. 
Journal of Atmospheric and Terrestrial Physics 53, 351-368.

${ }^{64}$ Omura, Y., Miyashita, Y., Yoshikawa, M., Summers, D., Hikishima, M., Ebihara, Y., Kubota, Y., 2015. J. Geophys. Res. 120, 9545-9562. doi:10.1002/2015JA021563.

${ }^{65}$ Sagdeev, R.Z., Usikov, D.A., Zaslavsky, G.M., 1988. Nonlinear Physics. From the Pendulum to Turbulence and Chaos.

${ }^{66}$ Shaaban, S.M., Lazar, M., Yoon, P.H., Poedts, S., López, R.A., 2019. Monthly Notices of the Royal Astronomical Society 486, 4498-4507. doi:10.1093/mnras/stz830, arXiv:1903.08005.

${ }^{67}$ Shklyar, D.R., 1981. Sov. Phys. JETP 53, 1197-1192.

${ }^{68}$ Shklyar, D.R., Matsumoto, H., 2009. Surveys in Geophysics 30, 55-104. doi:10.1007/s10712-009-9061-7.

${ }^{69}$ Shprits, Y.Y., Subbotin, D.A., Meredith, N.P., Elkington, S.R., 2008. Journal of Atmospheric and Solar-Terrestrial Physics 70, 1694-1713. doi:10.1016/j.jastp.2008.06.014.

${ }^{70}$ Solovev, V.V., Shkliar, D.R., 1986. Sov. Phys. JETP 63, 272277.

${ }^{71}$ Stenzel, R.L., 1999. J. Geophys. Res. 104, 14379-14396. doi: 10.1029/1998JA900120.

${ }^{72}$ Stix, T.H., 1962. The Theory of Plasma Waves.

${ }^{73}$ Summers, D., Thorne, R.M., Xiao, F., 1998. J. Geophys. Res. 103, 20487-20500. doi:10.1029/98JA01740.

${ }^{74}$ Tao, X., Bortnik, J., Albert, J.M., Thorne, R.M., 2012a. J. Geophys. Res. 117, 10205. doi:10.1029/2012JA017931.

${ }^{75}$ Tao, X., Bortnik, J., Thorne, R.M., Albert, J.M., Li, W., 2012 b. Geophys. Res. Lett. 39, 6102. doi:10.1029/2012GL051202.

${ }^{76}$ Tao, X., Chen, L., Liu, X., Lu, Q., Wang, S., 2017a. Geophysical Research Letters URL: http://dx.doi.org/10.1002/ 2017GL074881, doi:10.1002/2017GL074881.

${ }^{77}$ Tao, X., Zonca, F., Chen, L., 2017b. Geophys. Res. Lett. 44, 3441-3446. URL: http://dx.doi.org/10.1002/2017GL072624, doi:10.1002/2017GL072624.

${ }^{78}$ Thorne, R.M., 2010. Geophys. Res. Lett. 372, 22107. doi: 10.1029/2010GL044990.

${ }^{79}$ Thorne, R.M., Li, W., Ni, B., Ma, Q., Bortnik, J., Chen, L., Baker, D.N., Spence, H.E., Reeves, G.D., Henderson, M.G., Kletzing, C.A., Kurth, W.S., Hospodarsky, G.B., Blake, J.B., Fennell, J.F., Claudepierre, S.G., Kanekal, S.G., 2013. Nature 504, 411-414. doi:10.1038/nature12889.

${ }^{80}$ Thorne, R.M., Ni, B., Tao, X., Horne, R.B., Meredith, N.P., 2010. Nature 467, 943-946. doi:10.1038/nature09467.

${ }^{81}$ Titova, E.E., Kozelov, B.V., Jiricek, F., Smilauer, J., Demekhov, A.G., Trakhtengerts, V.Y., 2003. Annales Geophysicae 21, 1073-1081. doi:10.5194/angeo-21-1073-2003.

${ }^{82}$ Tong, Y., Vasko, I.Y., Artemyev, A.V., Bale, S.D., Mozer, F.S., 2019. Astrophys. J. 878, 41. doi:10.3847/1538-4357/ab1f05, arXiv: 1905.08958.

${ }^{83}$ Tyler, E., Breneman, A., Cattell, C., Wygant, J., Thaller, S., Malaspina, D., 2019. Geophys. Res. Lett. 46, 2328-2336. doi: 10.1029/2019GL082292.

${ }^{84}$ Vainchtein, D., Zhang, X.J., Artemyev, A., Mourenas, D., An- gelopoulos, V., Thorne, R.M., 2018. J. Geophys. Res. doi: 10.1029/2018ja025654.

${ }^{85}$ Van Compernolle, B., An, X., Bortnik, J., Thorne, R.M., Pribyl, P., Gekelman, W., 2015. Physical Review Letters 114, 245002. doi:10.1103/PhysRevLett.114.245002.

${ }^{86}$ Van Kampen, N.G., 2003. Stochastic Processes in Physics and Chemistry. 3 ed., North Holland.

${ }^{87}$ Vasiliev, A., Neishtadt, A., Artemyev, A., 2011. Physics Letters A 375, 3075-3079. doi:10.1016/j.physleta.2011.06.055.

${ }^{88}$ Vasko, I.Y., Mozer, F.S., Krasnoselskikh, V.V., Artemyev, A.V., Agapitov, O.V., Bale, S.D., Avanov, L., Ergun, R., Giles, B., Lindqvist, P.A., Russell, C.T., Strangeway, R., Torbert, R., 2018. Geophys. Res. Lett. 45, 5809-5817. doi: 10.1029/2018GL077835.

${ }^{89}$ Vedenov, A.A., Sagdeev, R.Z., 1961. Some properties of a plasma with an anisotropic ion velocity distribution in a magnetic field, in: Leontovich, M.A. (Ed.), Plasma Physics and the Problem of Controlled Thermonuclear Reactions, Volume 3, p. 332 .

${ }^{90}$ Vedenov, A.A., Velikhov, E., Sagdeev, R., 1962. Quasilinear theory of plasma oscillations. Nuclear Fusion Suppl. 2, 465475.

${ }^{91}$ Veltri, P., Mangeney, A., Scudder, J.D., 1992. Nuovo Cimento C Geophysics Space Physics C 15, 607-619. doi: 10.1007/BF02507834.

92 Veltri, P., Zimbardo, G., 1993. J. Geophys. Res. 98, 1333513346. doi:10.1029/93JA01144.

${ }^{93}$ Volokitin, A.S., Krafft, C., 2001a. Physics of Plasmas 8, 37483758. doi:10.1063/1.1380694.

${ }^{94}$ Volokitin, A.S., Krafft, C., 2001b. Physics of Plasmas 8, 49604971. doi:10.1063/1.1398085.

${ }^{95}$ Wilson, III, L.B., Cattell, C.A., Kellogg, P.J., Wygant, J.R., Goetz, K., Breneman, A., Kersten, K., 2011. Geophys. Res. Lett. 38, 17107. doi:10.1029/2011GL048671.

${ }^{96}$ Wilson, III, L.B., Koval, A., Szabo, A., Breneman, A., Cattell, C.A., Goetz, K., Kellogg, P.J., Kersten, K., Kasper, J.C., Maruca, B.A., Pulupa, M., 2012. Geophys. Res. Lett. 39, 8109. doi:10.1029/2012GL051581.

${ }^{97}$ Yoon, P.H., Pandey, V.S., Lee, D.H., 2013. Physics of Plasmas 20, 112902. doi:10.1063/1.4831965.

${ }^{98}$ Zhang, X., Angelopoulos, V., Artemyev, A.V., Liu, J., 2018a. Geophys. Res. Lett. 45, 9380-9389. doi:10.1029/2018GL079613.

${ }^{99}$ Zhang, X.J., Mourenas, D., Artemyev, A.V., Angelopoulos, V., Bortnik, J., Thorne, R.M., Kurth, W.S., Kletzing, C.A., Hospodarsky, G.B., 2019. Geophysical Research Letters 46, 71827190. URL: https://agupubs.onlinelibrary.wiley.com/doi/ abs/10.1029/2019GL083833, doi:10.1029/2019GL083833,

${ }^{100}$ Zhang, X.J., Thorne, R., Artemyev, A., Mourenas, D., Angelopoulos, V., Bortnik, J., Kletzing, C.A., Kurth, W.S., Hospodarsky, G.B., 2018b. J. Geophys. Res. 123, 53795393. URL: https://agupubs.onlinelibrary.wiley.com/doi/ abs/10.1029/2018JA025390, doi:10.1029/2018JA025390, 